\title{
GCU
}

Glasgow Caledonian

University

University for the Common Good

\section{Associations of intention to undertake physical activity among community dwelling British South Asian adults aged 60 years and over: a cross-sectional study}

Horne, M.; Emsley, R.; Woodham, A.; Wearden, A.; Skelton, D.A.

Published in:

Public Health

DOI:

10.1016/j.puhe.2018.05.005

Publication date:

2018

Document Version

Author accepted manuscript

Link to publication in ResearchOnline

Citation for published version (Harvard):

Horne, M, Emsley, R, Woodham, A, Wearden, A \& Skelton, DA 2018, 'Associations of intention to undertake physical activity among community dwelling British South Asian adults aged 60 years and over: a cross-sectional study', Public Health, vol. 162, pp. 1-8. https://doi.org/10.1016/j.puhe.2018.05.005

\section{General rights}

Copyright and moral rights for the publications made accessible in the public portal are retained by the authors and/or other copyright owners and it is a condition of accessing publications that users recognise and abide by the legal requirements associated with these rights.

Take down policy

If you believe that this document breaches copyright please view our takedown policy at https://edshare.gcu.ac.uk/id/eprint/5179 for details

of how to contact us. 


\section{Associations of intention to undertake physical activity among community dwelling}

British South Asian adults aged 60 and over: a cross sectional study.

Dr Maria Horne ${ }^{1 *}$, Dr Emsley ${ }^{2}$, Adrine Woodham ${ }^{3}$, Professor A Wearden ${ }^{4}$ and Professor DA Skelton ${ }^{5}$

${ }^{1}$ Faculty of Medicine and Health, School of Healthcare, University of Leeds, Leeds, LS2 9JT. Email: M.Horne@leeds.ac.uk

${ }^{2}$ Department of Biostatistics and Health Informatics, Institute of Psychiatry, Psychology and Neuroscience, King’s College London, WC2R 2LS. Email: richard.emsley@kcl.ac.uk

${ }^{3}$ Health Sciences Group - Primary Care, Institute of Population Health, The University of Manchester, University Place, Oxford Road, Manchester, UK M13 9PL.

Email: adrine.woodham@manchester.ac.uk

${ }^{4}$ School of Psychological Sciences, The University of Manchester, Oxford Road, Manchester, UK M13 9PL. Email: alison.wearden@manchester.ac.uk

${ }^{5}$ Institute of Applied Health Research, School of Health and Social Care, Glasgow Caledonian University, Glasgow, UK G4 0HB. Email: dawn.skelton@gcal.ac.uk

*Corresponding author: Dr Maria Horne,

Faculty of Medicine and Health,

School of Healthcare,

Baines Wing,

University of Leeds,

Leeds, LS2 9JT.

Tel: +44 (0)113 3431230

Email: M.Horne@leeds.ac.uk

ORCID ID: http://orcid.org/0000-0002-6153-8547 


\title{
Associations of intention to undertake physical activity among community dwelling British South Asian adults aged 60 and over: a cross sectional study.
}

\author{
ABSTRACT \\ OBJECTIVE: High prevalence of sedentary behaviour is seen in most immigrant groups in Western \\ countries, particularly in those from the Indian subcontinent. The primary objective of this study was \\ to determine the socio-cognitive associations with intention to undertake physical activity (PA) \\ among separate groups of Indian, Pakistani and Bangladeshi older adults aged $\geq 60$ in the North West \\ of England, UK.
}

STUDY DESIGN: Cross-sectional survey.

METHODS: Using a cross-sectional survey, informed by the Theory of Planned Behaviour (TPB), 138/964 South Asian (SA) older adults completed a mailed questionnaire that assessed self-reported PA and TPB variables.

RESULTS: Associations were seen in beliefs about PA and the amount of PA undertaken (rho=0.18, $\mathrm{p}=0.04)$. Attitude was correlated with intention ( $(\mathrm{hho}=0.29, \mathrm{p}=0.01)$ and amount of PA undertaken (rho=0.21, $\mathrm{p}=0.02$ ). Subjective norm was correlated with intention to undertake PA (rho=0.21, $\mathrm{p}=0.02$ ). Between-group differences were seen between: (1) Indian and Pakistani group's in subjective norm (2) all ethnicities in intention to undertake PA and (3) in the amount and attitude to undertaking PA between men and women. Co-morbidity was associated with beliefs about PA uptake. CONCLUSIONS: Subgroup differences between Indian, Pakistani and Bangladeshi older adults in subjective norm and intention to undertaking PA highlight the importance of tailoring interventions to accommodate for subgroup differences when developing culturally appropriate PA interventions aimed at increasing uptake and adherence.

\section{Keywords}

Physical activity; Older adults; Ethnicity; South Asian; Theory of Planned Behaviour. 


\section{Introduction}

Increasing physical activity (PA) levels across the lifespan is a major public health challenge. $60 \%$ of the world's population does not achieve the recommended minimum of 150 minutes of moderateintensity PA over-the-week [1]. In England, only 19\% of people aged 65-74 and 6\% of people aged 75+ meet recommended PA targets [2]. PA levels are lower amongst Black and Minority Ethnic (BME) groups, particularly South Asian (SA) older adults (those originating from the countries of Bangladesh, India and Pakistan). Compared with European whites, British SA adults are $60 \%$ less likely to meet recommended PA targets [3] with only $11 \%$ of SA men and $8 \%$ of SA women aged 55+ undertaking recommended levels of PA [4], but are at increased risk of stroke and type-2 diabetes [5].

Increasing PA among SA older adults can be difficult as a number of generic and sociocultural barriers exist. For example, many SA men and women report generic barriers such as cost, personal safety, lack of time outside of long working hours and carer commitments as negatively influencing PA uptake and maintenance [6,7]. Complex cultural beliefs about family roles/expectations, religious requirement for modesty, cultural rejection of a "sporting” identity or dress, language barriers and health knowledge can make becoming more physically active challenging $[6,8]$.

PA is also influenced by social-cognitive determinants. Unwillingness to participate in PA is related to beliefs about and attitudes to PA and to perceived behavioural control (PBC). PBC refers to a person's confidence in their ability to carry out the intended action, and may be influenced by perceived barriers to that action. To understand and explain PA intention and behaviour, the Theory of Planned Behaviour (TPB) is frequently used and its predictive value is well-established [9]. The TPB explains the process through which people change their behaviour, providing a useful starting point to identify possible behaviour change mechanisms, and how these can be addressed, when developing interventions [10]. The TPB highlights the importance of beliefs in forming intention to change health-related behaviour and states that PA among adults is predicted by their intention to undertake PA. Behavioural intention can be explained by three social-cognitive determinants - attitude, 
subjective norm, and PBC [10]. Attitude towards behaviour is determined by beliefs about the consequences of adopting that behaviour and is determined by behavioural beliefs, i.e. perceptions regarding the advantages/disadvantages of the behaviour. Subjective norm refers to the perceived social approval for the behaviour, determined by expectations regarding whether important reference individuals or groups will approve of the behaviour.

SA subgroups vary in terms of religion, culture and language, and demonstrate a mixed profile of health behaviours, including PA [3]. Research relating to ethnicity and health needs to examine diversity within BME groups to avoid homogenization [11]. Whilst studies have investigated the determinants of PA behaviour among SA older adults [6], none have explored subgroup variations using the TPB. To enable the development of effective PA interventions, tailored to the needs of Indian, Pakistani and Bangladeshi older adults, it is important to understand what determines PA intention in each subgroup. Therefore, this study aimed to determine the socio-cognitive associations of intention to undertake PA among separate groups of Indian, Pakistani and Bangladeshi older adults aged $\geq 60$ using the TPB.

\section{Methods}

Participants were recruited from ten general practices in the North West of England, UK. Given that minority groups are less likely to respond to surveys [12], we purposively sampled practices with a greater number of people from SA ethnic groups than in the general population. Patients who were terminally ill, had severe mental illness, had moderate to severe dementia, had moved away or were recently deceased were excluded.

The questionnaire was developed from previous qualitative work exploring SA older adult's culturally-determined beliefs in relation to PA, using strategies promoted by Ajzen [13] for construction and presentation. Hunt and Bhopal's [14] translation or adaptation procedures was used to ensure cultural appropriateness and substitution of English words with non-English words. For example, translation of items was conducted with bilinguals and monolingual people in the target languages and consulted on suitable word replacements were there was no English substitute e.g. the 
word 'joints' was translated to 'bones'. The questionnaire was pilot-tested in English, Urdu, Guajarati, Hindi, Punjabi, and Bangla and necessary revisions were made before finalising the survey. The survey assessed attitudes, beliefs, subjective norm and PBC in relation to undertaking and continuing with PA. Participants were asked their gender, ethnicity, marital status, year of birth, and how often they undertook PA.

\section{Physical activity measures}

Participants completed the four item Godin questionnaire [15], which provides a measure of type, duration and intensity of leisure-time PA. It provides examples of mild, moderate and strenuous exercise and asks how many times per week the respondent undertakes 15 minutes of each exercise category. A score of less than 14 is indicative of insufficient PA.

Regular moderate intensity activity was defined as '150 minutes (2 1 1/2 hours) of moderate level activity over the week - e.g. brisk walking. Moderate level activity makes you warm and slightly breathless but you are still able to hold a conversation' and was explained to participants before beginning the questionnaire.

\section{Social-cognitive determinants of PA}

For each of the scales measuring social cognitive determinants of PA, items were summed to produce the construct score, with higher scores representing more positive attitude, more positive behavioural beliefs, stronger subjective norm, greater perceived behavioural control and stronger intention. Cronbach’s alpha for the sample was calculated for each scale.

Attitude was assessed with three items on a five point Likert scale: (i) 'improving the movement of my bones (joints) would be.....'. Response categories ranged from $1=$ extremely bad to $5=$ extremely good; (ii) ‘For me, losing weight would be....' . Response categories ranged from 1 = very useful to $5=$ not useful at all and (iii) 'Increasing my social networks would be...'. Response categories ranged from $1=$ very useful to $5=$ not useful at all. Cronbach's alpha was 0.51 . 
Behavioural beliefs were assessed with 4 items on a five point Likert scale (e.g. 'to do 150 minutes of moderate level activity over the week in the next month would improve my general health'. Response categories ranged from $1=$ extremely unlikely to $5=$ extremely likely). Cronbach’s alpha was 0.76 .

Subjective norm was assessed with 4 items on a five point Likert scale (e.g. 'my husband/wife thinks that I should do 150 minutes of moderate level activity over the week’. Response categories ranged from 1 = agree strongly to 5 disagree strongly). Cronbach’s alpha was 0.86 .

Perceived behavioural Control was assessed with 4 items on a five point Likert scale (e.g. 'for me to do 150 minutes of moderate level activity over the week in the next month would be...'. Response categories ranged from 1 = easy to 5 = difficult). Cronbach's alpha was 0.91 .

Intention to undertake PA was assessed with 6 items on a five point Likert scale (e.g. 'how likely are you to do 150 minutes of moderate level activity over the week in the next month’. Response categories ranged from $1=$ extremely unlikely to 5 = extremely likely). Cronbach’s alpha was 0.62.

\section{Procedure}

The study was approved by the North West Research Ethics Committee (12/NW/0586). Practice-staff from recruited GP surgeries generated a list of anonymised SA patients aged $\geq 60$ from the practice register, and screened out those meeting the predefined exclusion criteria listed above. An invitation letter, with the English version of the questionnaire, was sent through the GP practice inviting participants. A letter translated into each of the five languages (Urdu/Guajarati/Hindi/Punjabi/Bangla) was included with all mailings informing participants that they could request the survey in these languages or could complete the survey by interview if preferred, with a translator if necessary. This letter asked them to:

1. complete the questionnaire and return it in the self-addressed envelope 
2. indicate the language of preference for the questionnaire to be mailed to them and return it in the self-addressed envelope

3. indicate if they wish to complete the survey by interview and return it in the self-addressed envelope

Questionnaire packs, including a freepost return envelope, were sent by GP practices to all registered eligible patients. Up to two reminders were sent, one month apart.

The participant information sheet, included in the letter, stated that informed consent would be assumed from completion of the survey. The information sheet and survey were translated into the languages principally used by members of SA groups in the participating practices Urdu/Guajarati/Hindi/Punjabi/Bangla. Techniques that improve response rates, e.g. mailing a postcard reminder two-weeks later and mailing of a second questionnaire package [16] were undertaken.

A test re-test reliability study was conducted with $10 \%$ of survey responders, who were invited to complete the same questionnaire after 14 days, to estimate temporal stability and internal consistency.

\section{Statistical analyses}

Data were analysed using Stata 12.1. Age and gender was compared between responders and non-responders to assess possible response bias. Spearman correlations were used to test whether the TPB constructs (attitude, beliefs, subjective norm and perceived behavioural control) were associated with intentions to exercise and reported exercise behaviour. Linear regression was used to identify if gender, ethnicity and co-morbidity independently have an effect on PA behaviour, reported as between-group mean differences and their standard errors and 95\% confidence intervals. Missing items were prorated across the scale to account for missing item data if less than $50 \%$ of items were missing; otherwise they were treated as missing, and analysis carried out on remaining data.

\section{Results}


The survey was sent out to 972 SA people aged $\geq 60$ years. Screening excluded patients who were terminally ill, had severe mental illness, moderate-severe dementia, had moved away or were recently deceased, but 8 questionnaire packs were returned undelivered. From the resulting adjusted sample of 964 people, 138 people returned the questionnaire (14.3\% of the adjusted total), with 134 (13.9\% of the adjusted total) returning it fully completed. A summary of the demographics are shown in Table 1. We found that $55 \%$ of the responders were male. The majority were married (71\%) and largely of Pakistani ethnicity (75\%). Responders mean (SD) age was 68.9 (6.48). 43\% of the sample reported undertaking half-an-hour activity on 1-4 days per week over past month; the mean (SD) Godin exercise score was 13.6 (14.09).

$<$ Table 1 $>$

\section{Intention to undertake PA}

Table 2 shows correlations between the main TPB constructs, intention to undertake PA, and self-reported PA (Table 2). Only small correlations were seen between the amount of PA and intention to undertake PA. Attitude was correlated with intention to undertake PA (rho, 0.29, p = 0.01 ) and amount of PA (rho $=0.21, \mathrm{p}=0.02$ ), so that more positive attitudes were associated with greater intention and more PA. Subjective norm was correlated with intention to undertake PA (rho = $0.21, p=0.02$ ), so that stronger belief in the value of PA to significant others was associated with more PA.

$<$ Table 2>

\section{Between group differences of gender, ethnicity and co-morbidity}

We found a difference in attitude to undertake PA between men and women, where men held a more positive attitude to undertaking PA, but there was no gender difference in intention to 
undertake PA (Table 3). Differences were also seen in the amount of PA undertaken between men and women with men undertaking more PA than women.

There were differences in subjective norm between Indian and Pakistani older adults and between all ethnic groups in intention to exercise; Bangladeshi older adults had the highest intention and Indian older adults had the lowest intention (Table 3).

$<$ Table 3>

Co-morbidity influenced beliefs about participants’ ability to undertake PA; those with 3 and more comorbidities were less likely to believe they could undertake PA.

\section{Correlation between PA and individual items in the attitude scale by ethnicity}

On the attitudinal subscale, we found correlations between attitude to undertaking PA to improve movement of joints (rho $=0.50, \mathrm{p}=0.05)$ and to lose weight $($ rho $=-0.49, \mathrm{p}=0.05)$ and amount of PA of Indian ethnicity. A correlation was seen in attitude to undertaking PA to improve social networks (rho $=0.22, \mathrm{p}=0.03$ ) and amount of PA in participants of Pakistani ethnicity. There were no significant correlations between individual items on the attitude scale and PA among participants of Bangladeshi ethnicity.

\section{$<$ Table 4>}

\section{Discussion}

This study investigated ethnicity differences among SA older adults intention to undertake PA and self-reported PA using the TPB. However, this study found that intention did not correlate with the amount of PA undertaken and finds support in the literature, which highlights the poor translation of behavioural intentions into behaviour [17]. The use of implementation intentions, or 'If-Then' plans, 
have been found to improve rates of intention-behaviour translation [18] and offer a potentially effective and inexpensive intervention to assist people to act on their positive intentions [19].

Overall, attitude and subjective norm were the strongest constructs associated with PA levels, but differences existed between participants in the different ethnic subgroups. Additionally, increasing number of co-morbidities and female were associated with lower levels of PA. Subjective norm was associated with intention to undertake PA only amongst Pakistani older adults, who appear to be influenced more so by social pressure. Similarly, attitude to undertaking PA to improve social networks was only associated in participants of Pakistani ethnicity.

Although, subjective norm has been found to be a significant predictor of intention in similar research with ethnicity using the TPB [20], we identified subgroup differences. Generally, collectivist societies, like those from South Asia, tend to assign greater weight to social considerations (which may be reflected in the measure of subjective norm) when making decisions to act [20]. Although intention to participate in PA among older SA adults is generally influenced by social considerations, this was found not to be the case for those of Indian and Bangladeshi ethnicity in this study. Indeed, Indians had positive attitudes regarding PA to improve bone/joint function and lose weight, suggesting physical health improvement may be motivational to them. It has been argued that cultures do not align to a single dimension from individualism to collectivism [21] and that the degree to which the cultures in the country of origin are adhered to in the UK may be influenced by acculturation and cultural identity [22]. This may provide an explanation for the differences seen between these subgroups.

The presence of gender differences in uptake is consistent with most prior research amongst this population group [23]. Gender is known to be an important predictor of intention to uptake PA [24], with cultural expectations, norms and family obligations having a stronger influence on SA women undertaking and maintaining regular PA [25]. The differences seen in this study could be a consequence of PA reporting accuracy among SA women and that in SA culture, a woman's focus is on the family and domestic duties take priority over all other activities [26]. Therefore, much of their PA may be domestic activity related, which is not included in the Godin questionnaire or considered 
as moderate intensity activity in our questionnaire, so their level of PA may have been underestimated. Studies using objective monitoring of PA, not subjective self-report, have found that a higher percentage of SA women achieve the PA recommendations than would have been expected based on studies using self-reported PA [27].

The correlation between increasing co-morbidities and less positive beliefs about undertaking PA is consistent with prior research among other ethnic groups [28], and appears to be related to misunderstandings about the value of PA in later life among those with ongoing health problems [28]. Co-morbidity influences beliefs about the capacity of older adults to undertake PA; fear that it could exacerbate pre-existing health problems, result in physical harm [29] or feel they do not have as much control over their ability to participate in PA [30]. Therefore, those with co-morbidities are less inclined to take-up regular PA. Healthcare professionals may need to challenge misguided assumptions about PA and raise awareness about the beneficial effects of regular PA in ameliorating metabolic and psychological comorbidities [31] to positively influence attitudes to undertaking PA [32].

To our knowledge, in the context of the TPB, there are no published studies considering SA older adults, making it difficult to place findings in perspective, but highlights the value of this work in contributing to the knowledge base. TPB studies conducted in the field of PA among other ethnic groups have found ethnicity to be a moderating influence on attitude and exercise-intention [33]. Some differences were seen between all ethnicities in intention to undertake PA, which has implications for PA intervention research and supports the development of a toolkit of adaptation approaches for tailoring PA interventions, acknowledging subtle individual and subgroup ethnic differences.

Although this study provides important information about the associations of PA uptake among SA older adults, some limitations need to be considered when interpreting the results. The main limitation of this study was the low response rate and the resultant limitations this has placed on robust interpretation of the subgroup analysis. In particular, we had a very low response rate within the Indian and Bangladeshi population. The percentages of non-responders and responders from the 
total sample were the same, but it is likely that co-morbidities and PA behaviour and intention would be different in non-responders, with responses from people who are more likely to appreciate the benefit of PA. The problem of non-response may have biased our findings in relation to ethnic group, since selection bias may potentially have operated within these groups [34]. However, BME groups have lower response rates in almost all Western countries [35]. Moreover, the recording of ethnicity data in NHS records was not consistent across the sites, making it difficult to identify the ethnic group of all non-responders. NHS-recorded ethnicity is generally concordant with a person's self-identified ethnic group provided that person is from a White British background, but this is often not the case for people from BME groups [36]. We have no explanation for the striking feature of zero responses from the ‘Unknown Ethnic Group’. As ethnicity is usually well captured within GP databases, this group may not have been normal patients in GP surgeries; perhaps they were visitors and were added to the database with a view to not being followed up. Alternatively, these people did not want to give their ethnicity originally and perhaps likewise do not want to take part in surveys. In the future, this low response rate could potentially be improved by asking local community elders to distribute surveys rather than using GP databases, however, this will also result in bias in respondents.

Secondly, PA was based on self-report measures, not objectively measured, and could be influenced by what the individuals perceive as good/poor PA levels and subject to recall bias. Therefore, generalisation of these results is limited warranting further research in this area to understand subgroup differences in PA. Nevertheless, the survey results have provided some insight into subgroup variation in intention to undertake PA. Thirdly, the internal consistency (Cronbach's alpha) for the attitude measurement in our sample was only 0.51 , which is considered low. This implies that the average inter-item covariance among the items was low, though this is also reflective of the small number of items. A final limitation is the cross sectional nature of the study. Intention was the outcome variable and not prospective behaviour. Although intention is an important determinant of future PA behaviour [11], it remains to be determined if intention is a significant determinant of behaviour for this population group. Nonetheless, this study still provides an important contribution to our understanding of the link between SA older adults' intention and uptake of PA. 
Despite the given limitations, the findings of this study add to the knowledge base. Few studies have investigated the contributing factors to PA uptake among SA older adults; none have looked at subgroup variations using the TPB. Differences were seen between Bangladeshi, Indian and Pakistani older adults in intention to undertake PA. Subjective norm was associated with intention to undertake PA only among Pakistani’s. Likewise, attitude to undertaking PA to improve social networks was only associated in participants of Pakistani ethnicity, representing a new contribution to the literature.

\section{Conclusions}

PA interventions targeting a mainly Pakistani population group should consider family, peer and community involvement in PA support. Further research is needed to better understand subgroup variations with larger sample sizes to address the heterogeneity found within SA groups in this study, who may have different motivations to undertaking and increasing their PA levels.

\section{Author statements}

\section{Ethical approval}

The study was approved by the North West Research Ethics Committee (12/NW/0586).

\section{Funding}

The research project was funded by the NIHR Greater Manchester Collaboration for Leadership in Applied Health Research and Care (CLAHRC), RCF_R9_MH. The funding body had no input to the design of the study and collection, analysis, and interpretation of data or in writing the manuscript.

\section{Competing interests}

None declared. 


\section{References}

[1] World Health Organization. Global recommendations on physical activity for health. Geneva: World Health Organization, 2010.

[2] NHS Information Centre. Health Survey for England Adult Trend Tables 2007. London: NHS Information Centre, 2008.

[3] Willimas ED, Nazroo JY, Kooner JS and Steptoe A. Subgroup differences in psychosocial factors relating to coronary heart disease in the UK South Asian population. Journal of Psychosomatic Research 2010; 69(4-3): 379-387.

[4] Sproston K and Mindell J (eds). Health Survey for England 2004: The Health of Minority Ethnic Groups. London: Information Centre, 2006.

[5] Yates T, Davies MJ, Gray LJ, Webb D, Henson J, Gill JM, Sattar N and Khunti K. Levels of physical activity and relationship with markers of diabetes and cardiovascular disease risk in 5474 white European and South Asian adults screened for type 2 diabetes. Preventive Medicine 2010: 51(34): $290-4$.

[6] blinded for peer review.

[7] Cross-Bardell L., George T, Bhoday M., Tuomainen H, Qureshi N and Kai J. Perspectives on enhancing physical activity and diet for health promotion among at-risk urban UK South Asian communities: a qualitative study. BMJ Open 2015; 5: e007317.

[8] Grace C, Begum R, Subhani S, Kopelman P. \& Greenhalgh T. Prevention of type 2 diabetes in British Bangladeshis: qualitative study of community, religious, and professional perspectives. British Medical Journal 2008, 337: a1931.

[9] Hagger MS, Chatzisarantis NLD and Biddle SJH. A meta-analytic review of the theories of reasoned action and planned behaviour in physical activity: predictive validity and the contribution of additional variables. Journal of Sport and Exercise Psychology 2002; 24(1): 3-32.

[10] Ajzen I. The theory of planned behaviour. Organizational Behavior and Human Decision Processes 1991; 50(2): 179-211. 
[11] Mir G, Salway S, Kai J, Karlsen S, Bhopal R., Ellison GTH and Sheikh A. Principles for research on ethnicity and health: the Leeds Consensus Statement. European Journal of Public Health 2012; 23(3): 504-510.

[12] Sheldon H, Graham C, Pothecary N and Rasul F. Increasing response rates amongst Black and minority ethnic and seldom heard groups. Picker Institute Europe, 2007. Available from: http://www.nhssurveys.org/Filestore/documents/Increasing_response_rates_literature_review.p $\underline{\mathrm{df}}$ Accessed 20 $0^{\text {th }}$ November 2017.

[13] Ajzen I. Constructing a TPB Questionnaire: Conceptual and Methodological Considerations, 2002 [Website], Available from: https://people.umass.edu/aizen/pdf/tpb.measurement.pdf Accessed 20 $0^{\text {th }}$ November 2017.

[14] Hunt S and Bhopal R. Self report in clinical and epidemiological studies with non-English speakers: the challenge of language and culture. Journal of Epidemiology and Community Health, 2004; 58(7): 618-22.

[15] Godin G and Shephard RJ. Godin Leisure-Time Exercise questionnaire. Medicine and Science in Sports and Exercise 1997; 29 (suppl.), S36-S38.

[16] McColl, E., Jacoby, A., Thomas, L., Soutter, J. Bamford, C., Steen, N. ... and Bond, J. Design and use of questionnaires: a review of best practice applicable to surveys of health service staff and patients. Health Technology Assessment 2001; 5: 31.

[17] Sheeran P. Intention-behaviour relations: A conceptual and empirical review. European Journal of Social Psychology 2002; 12:1-36.

[18] Ziegelmann JP, Luszczynska A, Lippke S and Schwarzer R (2007). Are goal intentions or implementation intentions better predictors of health behavior? A longitudinal study in orthopaedic rehabilitation. Rehabilitation Psychology 2007; 52: 97-102.

[19] Gollwitzer PM. Implementation intentions: Strong effects of simple plans. American Psychologist 1999; 54:493-503 
[20] Hagger MS, Chatzisarantis NLD, Barkoukis V, Wang JC, Hein V, Pihu M, Soos I and Karsai I. Cross-cultural generalizability of the Theory of Planned Behavior among young people in a physical activity context. Journal of Sport and Exercise Psychology 2007; 29(1): 2-20.

[21] Knight, B. G. and Sayegh, P. Cultural values and caregiving: the updated sociocultural stress and coping model. Journals of Gerontology: Psychological Sciences \& Social Sciences 2009; 65B (1): 5-13.

[22] Burholt V, Dobbs C and Victor C. Social support networks of older migrants in England and Wales: the role of collectivist culture. Ageing \& Society 2017: 1-25.

[23] Williams ED, Stamatakis E, Chandola T and Hamer M. Assessment of physical activity levels in South Asians in the UK: findings from the Health Survey for England. Journal of Epidemiology and Community Health 2011; 65 (6): 517-521.

[24] Babkus WS and Thompson JL. Physical activity among South Asian women: a systematic, mixed-methods review. International Journal of Behavioral Nutrition and Physical Activity 2012; 9:150.

[25] Koshoedo SA, Paul-Ebhohimhen VA, Jepson RG and Watson MC. Understanding the complex interplay of barriers to physical activity amongst black and minority ethnic groups in the United Kingdom: a qualitative synthesis using meta-ethnography. BMC Public Health 2015; 15: 643. [26] Sriskantharajah J, Kai J. Promoting physical activity among South Asian women with coronary heart disease and diabetes: what might help? Family practice 2007;24(1):71-6.

[27] Curry WB and Thompson JL. Objectively measured physical activity and sedentary time in south Asian women: a cross-sectional study. BMC Public Health 2014: 14: 1269.

[28] Putcha N, Han MK, Martinez CH et al. Comorbidities of COPD have a Major Impact on Clinical Outcomes, Particularly in African Americans. Chronic Obstructive Pulmonary Diseases: Journal of the COPD Foundation 2014; 1: 105-114.

[29] blinded for peer review. 
[30] Brassington GS, Atienza AA, Perczek RE, DiLorenzo TM and King AC. Intervention-related cognitive versus social mediators of exercise adherence in the elderly. American Journal of Preventive Medicine 2002; 23 (2 Suppl): 80-86.

[31] blinded for peer review.

[32] Tsatsoulis A and Fountoulakis S. The protective role of exercise on stress system dysregulation and comorbidities. Annals of the New York Academy of Sciences 2006; 1083: 196-213.

[33] Blanchard CM, Rhodes RE, Nehl E, Fischer J, Sparling P and Courneya KS. Ethnicity and the Theory of Planned Behavior in the exercise domain. Amercican Journal of Health Behavior 2003; 27(6): 579-591.

[34] Feskens RCW, Hox J, Lensvelt-Mulders G and Schmeets H. Nonresponse among ethnic minorities: A multivariate analysis. Journal of Official Statistics 2007; 23(3): 387-408.

[35] Feskens RCW, Hox J, Lensvelt-Mulders G and Schmeets H. Collecting data among ethnic minorities in an international perspective. Field Methods 2006; 18(3): 284-304.

[36] Saunders K. Do NHS records reflect patient ethnicity? 2014 [website]. Available from: http://www.statslife.org.uk/health-medicine/1940-do-nhs-records-reflect-patient-ethnicity Accessed 20 $0^{\text {th }}$ November 2017. 
Table 1: Characteristics of Sample

\begin{tabular}{|c|c|c|c|}
\hline $\begin{array}{l}\text { Demographic } \\
\text { Variables }\end{array}$ & $\begin{array}{c}\text { Responders } \\
\mathrm{N}=138\end{array}$ & $\begin{array}{c}\text { Non-responders } \\
\mathrm{N}=834\end{array}$ & Comparison \\
\hline \multicolumn{4}{|l|}{ Ethnicity: N (\%) } \\
\hline Indian & $19(14)$ & $90(11)$ & - \\
\hline Pakistani & $103(75)$ & $477(57)$ & \\
\hline Bangladeshi & $9(7)$ & $46(6)$ & \\
\hline Other & 7 (5) & 0 & \\
\hline Unknown & & $221(27)$ & \\
\hline \multicolumn{4}{|l|}{ Gender: N (\%) } \\
\hline Male & $76(55)$ & $414(50)$ & $X^{2}=1.60$ \\
\hline Female & $61(45)$ & $420(50)$ & $P=0.206$ \\
\hline $\begin{array}{l}\text { Age (years): Mean } \\
\text { (SD) }\end{array}$ & $68.90(6.48)$ & $69.06(7.67)$ & $\begin{array}{l}T=-0.232 \\
P=0.8168\end{array}$ \\
\hline \multicolumn{4}{|l|}{ Marital Status: N (\%) } \\
\hline Married & $96(71)$ & NA & \\
\hline Divorced & $4(3)$ & & \\
\hline Widowed & $31(23)$ & & \\
\hline Other & $5(3)$ & & \\
\hline Missing & 3 & & \\
\hline \multicolumn{4}{|l|}{ Physical activity: } \\
\hline $\begin{array}{l}\text { Over past month, how } \\
\text { many days in a week } \\
\text { with half an hour } \\
\text { activity? }\end{array}$ & & NA & \\
\hline$N(\%)$ & $36(27 \%)$ & & \\
\hline 0 days per week & $57(43 \%)$ & & \\
\hline 1-4 days per week & $16(12 \%)$ & & \\
\hline 5 days per week & $24(18 \%)$ & & \\
\hline 6-7 days per week & 5 & & \\
\hline Missing & & & \\
\hline \multicolumn{4}{|l|}{ Co-morbidities: N (\%) } \\
\hline & $54(46)$ & NA & \\
\hline $3+$ & $63(54)$ & & \\
\hline Missing & 21 & & \\
\hline $\begin{array}{l}\text { Godin Score: } \\
\text { Mean (SD) }\end{array}$ & $13.56(14.09)$ & NA & \\
\hline
\end{tabular}


Table 2: Spearman correlation coefficients between intention and exercise and TPB constructs.

\begin{tabular}{llllll}
\hline & Beliefs & Attitude & $\begin{array}{l}\text { Subjective } \\
\text { norm }\end{array}$ & PBC & Intention \\
\hline Intention & -0.14 & 0.29 & 0.21 & -0.08 & - \\
& $(0.12)$ & $(<0.01)$ & $(0.02)$ & $(0.39)$ & \\
^Physical & 0.18 & 0.21 & -0.01 & -0.04 & 0.06 \\
Activity & $(0.04)$ & $(0.02)$ & $(0.90)$ & $(0.69)$ & $(0.48)$ \\
\hline
\end{tabular}

Spearman correlation ( $p$-value).

$\wedge$ Physical activity measured as numbers of days per week on which there was at least 30 minutes of moderate intensity activity 
Table 3: Between group differences of gender, ethnicity and co-morbidity on Theory of Planned Behaviour (TPB) constructs, intention to exercise and amount of physical activity.

\begin{tabular}{|c|c|c|c|c|c|c|}
\hline Predictors & Beliefs & Attitude & $\begin{array}{c}\text { Subjective } \\
\text { norm }\end{array}$ & PBC & Intention & $\begin{array}{l}\text { ^Physical } \\
\text { Activity }\end{array}$ \\
\hline Male & 14.27 & 9.63 & 10.55 & 12.52 & 16.22 & 1.40 \\
\hline Difference & $\begin{array}{l}-0.57(0.76) \\
(-2.05 \text { to } 0.91)\end{array}$ & $\begin{array}{l}-1.05(0.46) \\
(-1.95 \text { to }-0.14) \dagger\end{array}$ & $\begin{array}{l}-0.83(0.83) \\
(-2.46 \text { to } 0.80)\end{array}$ & $\begin{array}{l}0.19(0.34) \\
(-0.48 \text { to } 0.86)\end{array}$ & $\begin{array}{l}-0.49(0.69) \\
(-1.85 \text { to } 0.87)\end{array}$ & $\begin{array}{l}-0.43(0.17) \\
(-0.77 \text { to }-0.10) \dagger\end{array}$ \\
\hline \multicolumn{7}{|l|}{ Ethnicity } \\
\hline Indian (1) & 15.02 & 8.56 & 7.24 & 12.54 & 13.72 & 1.56 \\
\hline Other (4) & 15.00 & 9.83 & 8.33 & 11.5 & 16.50 & 1.00 \\
\hline Difference (1 vs. 2) & $\begin{array}{l}-1.43(0.95) \\
(-3.29 \text { to } 0.44)\end{array}$ & $\begin{array}{l}0.62(0.54) \\
(-0.44 \text { to } 1.68)\end{array}$ & $\begin{array}{l}3.67(0.86) \\
(1.98 \text { to } 5.35) \dagger\end{array}$ & $\begin{array}{l}0.16(0.52) \\
(-0.87 \text { to } 1.19)\end{array}$ & $\begin{array}{l}2.39(1.08), \\
(0.26 \text { to } 4.52) \dagger\end{array}$ & $\begin{array}{l}-0.44(0.28), \\
(-0.98 \text { to } 0.11)\end{array}$ \\
\hline & $\begin{array}{l}1.20(1.33) \\
(-1.41 \text { to } 3.81)\end{array}$ & $\begin{array}{l}1.44(1.06) \\
(-0.64 \text { to } 3.51)\end{array}$ & $\begin{array}{l}2.39(1.53) \\
(-0.61 \text { to } 5.40)\end{array}$ & $\begin{array}{l}-0.16(0.70) \\
(-1.53 \text { to } 1.20)\end{array}$ & $\begin{array}{l}5.53(1.35) \\
(2.88 \text { to } 8.18) \dagger\end{array}$ & $\begin{array}{l}0.11(0.37) \\
(-0.61 \text { to } 0.83)\end{array}$ \\
\hline \multicolumn{7}{|l|}{ Co-morbidity } \\
\hline $0-2$ & 14.81 & 9.57 & 9.97 & 12.48 & 15.40 & 1.35 \\
\hline $3+$ & 13.05 & 8.78 & 10.46 & 12.95 & 16.32 & 1.10 \\
\hline Difference & $\begin{array}{l}-1.75(0.76) \\
(-3.24 \text { to }-0.27) \dagger\end{array}$ & $\begin{array}{l}-0.79(0.46) \\
(-1.68 \text { to } 0.11)\end{array}$ & $\begin{array}{l}0.50(0.84) \\
(-1.15 \text { to } 2.14)\end{array}$ & $\begin{array}{l}0.46(0.38) \\
(-0.27 \text { to } 1.20)\end{array}$ & $\begin{array}{l}0.92(0.82) \\
(-0.70 \text { to } 2.54)\end{array}$ & $\begin{array}{l}-0.26(0.19) \\
(-0.62 \text { to } 0.11)\end{array}$ \\
\hline
\end{tabular}


$\wedge$ Physical activity measured as numbers of days per week on which there was at least 30 minutes of moderate intensity activity Results show means. Differences are between group mean differences (standard errors), (95\% confidence intervals).

†Significant difference at 0.05 significance level.

PBC - Perceived Behavioural Control. 
Table 4: Correlation between actual exercise and individual items in the attitude scale by ethnicity.

\begin{tabular}{lcccc}
\hline & Bones/Joints & $\begin{array}{c}\text { Losing } \\
\text { weight }\end{array}$ & $\begin{array}{c}\text { Social } \\
\text { networks }\end{array}$ & Too old \\
\hline Exercise & 0.50 & -0.49 & 0.21 & -0.06 \\
(Indian) & $(0.05)$ & $(0.05)$ & $(0.42)$ & $(0.86)$ \\
& 16 & 17 & 17 & 18 \\
Exercise & 0.10 & 0.14 & 0.22 & 0.35 \\
(Pakistani) & $(0.34)$ & $(0.18)$ & $(0.03)$ & $(<0.01)$ \\
& 96 & 99 & 96 & 96 \\
Exercise & 0.35 & 0.12 & 0.44 & -0.23 \\
(Bangladeshi) & $(0.36)$ & $(0.77)$ & $(0.28)$ & $(0.57)$ \\
& 9 & 9 & 8 & 9 \\
\hline
\end{tabular}

Spearman correlation ( $p$-value) 